\title{
Comparação da Aterosclerose Coronária em Pacientes com Infarto do Miocárdio e Angina do Peito
}

\author{
Waldomiro Carlos Manfroi, Alcides José Zago, Cristiane Bauermann Leitão, Karen Gomes Ordovás, \\ Letícia Weiss Ribeiro, Josiane de Souza, Luciana Kirschnick, Rafael Henriques Candiago, Rosana Cruz, \\ Alexandre Goellner, llza Dias
}

\author{
Porto Alegre, RS
}

\begin{abstract}
Objetivo-Comparar a gravidade da doença coronária e a presença de fatores de risco cardiovasculares entre pacientes com angina e infarto do miocárdio (IM).

Métodos - Estudaram-se 62 pacientes com IM 129 com angina, através de cineangiocoronariografia, avaliando-se a oclusão (lesão de $99 \%$ ou 100\%), a severidade (escore de 0 a 5 de acordo com o número de vasos afetados) e a extensão (3 grupos com diferentes graus de estenose). Dois observadores experientes interpretaram cegamente os angiogramas.

Resultados - Os pacientes com IM tiveram maior oclusão (50\% vs 13,2\% [p<0,01]), maior severidade (79\% vs $54,3 \%$ com mais de $90 \%$ de estenose $[p<0,02])$ e maior extensão (2,0 vs 0,87 [p<0,001]), mesmo quando controlados para os fatores de risco coronários clássicos e para o tempo de doença. O tabagismo foi o único fator de risco independente correlacionado com IM $(P<0,01)$.

Conclusão - Entre os pacientes estudados, a doença coronária foi maior no grupo IM, bem como a prevalência de tabagismo.
\end{abstract}

Palavras-chave: infarto do miocárdio, angina do peito, cineangiocoronariografia

\section{Comparison of Coronary Heart Disease Extent and Severity in Patients with Myocardial Infarction and Angina Pectoris}

Purpose - To compare the severity of the coronary heart disease and the presence of coronary risk factors between angina and myocardial infarction (MI) patients.

Methods - We studied 62 patients with MI and 129 with angina through coronary angiography to evaluate occlusion (lesion of $99 \%$ or 100\%), extent (with a score of 0-5 derived by the number of vessels affected) and severity (3 groups of different stenosis degrees). Two experient observers blindly interpretated the angiograms.

Results - Patients with MI had more occlusions $(50 \%$ vs $13.2 \%$ [p<0.01]), more severity (79\% vs $54.3 \%$ with $>90 \%$ stenosis $[p<0.02])$ and more extent $(2.0$ vs 0.87 $[p<0.001])$, even when controlled for current coronary risk factors and disease duration. Smoking was the only independent risk factor related to MI $(p<0.001)$.

Conclusion - Among the studied patients, coronary heart disease extent and severity was greater in the MI group, as well as the prevalence smoking.

Key-words: myocardial infarction, angina pectoris, coronary angiography

Arq Bras Cardiol, volume $71\left(n^{\circ} 1\right), 25-29,1998$

A doença isquêmica do coração e os acidentes vasculares cerebrais continuam sendo as causas mais freqüentes de morte nos países industrializados, ambos tendo como substrato etiopatogênico $\mathrm{a}$ aterosclerose ${ }^{1}$. Quanto à gênese da aterosclerose coronária, trabalhos de investigação realiza-

Hospital de Clínicas de Porto Alegre - UFRGS

Correspondência: Waldomiro Carlos Manfroi - Rua Ramiro Barcelos, 2350 - $2^{\circ}$ - S/2060 - 90035-003 - Porto Alegre, RS

Recebido para publicação em 14/7/97

Aceito em 20/3/98 dos durante décadas têm demonstrado tratar-se de uma proliferação de células musculares lisas, deposição de colesterol e infiltração de células mononucleares ${ }^{2}$. Por outro lado, estudos epidemiológicos realizados de forma sistemática identificaram vários fatores relacionados com a gravidade da doença, destacando-se entre eles a hipertensão arterial sistêmica (HAS), o tabagismo, a idade, o sexo, o diabetes mellitus, o número de artérias comprometidas e o comprometimento funcional do ventrículo esquerdo ${ }^{3}$. Ultimamente, outros estudos têm demonstrado ainda redução dos eventos agudos de isquemia miocárdica e de morte, quando são controlados 
esses fatores, estabelecendo uma relação entre a aceleração da aterosclerose e esses fatores de risco ${ }^{4,5}$.

Está estabelecido na literatura que as síndromes isquêmicas (angina estável, angina instável e infarto agudo do miocárdio (IAM)) possuem componente aterosclerótico em proporções variáveis, dentro do mesmo grupo populacional e em populações distintas, havendo inclusive um grupo de pacientes acometido por infarto do miocárdio (IM) com coronárias normais, como evidenciado em estudos previamente conduzidos por nosso grupo de pesquisas, bem como por outros autores ${ }^{6-11}$.

Estudos recentes demonstraram, ainda, que pacientes com IAM e angina instável apresentavam doença coronária menos extensa e menos grave do que pacientes com síndrome isquêmica crônica (angina estável). Esse achado foi atribuído à participação de fenômenos trombóticos e espasmódicos, como fatores precipitantes das síndromes isquêmicas agudas ${ }^{12,13}$. Aliado a este fato, Little e col observaram, através de cineangiocoronariografias seriadas, não ser possível afirmar que o segmento coronário mais acometido por aterosclerose seja o determinante de IM subseqüente ${ }^{14}$.

Por outro lado, não está bem estabelecido se algum dos fatores de risco tem participação independente na instabilidade da placa para o desencadeamento do IM ou se o mesmoé resultante do maior número de artérias comprometidas e maior gravidade das lesões.

A motivação para a realização deste estudo surgiu da impressão de um dos autores, durante 20 anos de realização de estudos cineangiocoronariográficos, de que o comprometimento anatômico das artérias coronárias seria semelhante em pacientes com e sem IM.

O objetivo deste estudo é avaliar, angiograficamente, se existe diferença quanto ao grau de obstrução e número de artérias coronárias comprometidas nos pacientes acometidos de IM, em relação aos pacientes com outras formas de manifestação de cardiopatia isquêmica, bem como investigar se existe participação dos fatores de risco no desenvolvimento do IM.

\section{Métodos}

Estudo transversal, incluindo 191 pacientes admitidos na Unidade de Hemodinâmica do Serviço de Cardiologia do Hospital de Clínicas de Porto Alegre (HCPA), de março/94 a janeiro/96, provenientes da internação ou ambulatório. Os pacientes foram selecionados de forma consecutiva, em função da disponibilidade dos pesquisadores, apresentando diagnóstico clínico de cardiopatia isquêmica e indicação formal de seu médico assistente para realização de cineangiocoronariografia. O grupo de pesquisa não participou da indicação do procedimento.

Os pacientes foram separados em dois grupos distintos: infarto - pacientes com IM, recente ou antigo, definido por história de dor típica associada a alteração eletrocardiográfica compatível, independente da presença de outra síndrome isquêmica prévia ou posterior ao episódio de IAM (n=62); angina - pacientes com manifestações clí- nicas compatíveis com angina estável ou angina instável, sem evidência de IM prévio na história e no eletrocardiograma $(n=129)$.

Antes de serem submetidos ao procedimento, os pacientes foram convidados a participar do estudo, assinando um termo de consentimento que autorizava a retirada de $10 \mathrm{~mL}$ de sangue para posterior análise laboratorial. A seguir, responderam a questionário padronizado especialmente elaborado para a investigação e identificação da presença ou não dos fatores de risco, situação clínica da doença e tempo de sua evolução.

Foi realizado estudo hemodinâmico e cineangiocardiográfico através de cateterismo cardíaco esquerdo, em que se dissecou a artéria braquial esquerda ou por punção da artéria femoral direita, sob ação de anestesia local (xilocaína 2\%). A cineangiocoronariografia seletiva seguiu a técnica de Sones ${ }^{15}$ ou Judkins ${ }^{16}$. Os filmes cineangiocardiogáficos foram interpretados por dois pesquisadores experientes, que desconheciam a presença ou não dos fatores de risco, bem como a história prévia ou não de IAM.

A amostra de sangue coletada foi analisada na Unidade de Bioquímica do HCPA, tendo sido feita a dosagem de colesterol total e triglicerídeos (método enzimático colorimétrico usando autoanalyser Selectra-Vitaldo, Merck), HDL e LDL (precipitação iônica seletiva/método enzimático colorimétrico), ferritina sérica (enzima imuno ensaio no equipamento Status Bayter), apolipoproteína A e apolipoproteína B (imunoturbidimetria e medição fotométrica com reagentes do kit Turbiquant).

Para quantificar a extensão e a severidade das estenoses arteriais coronárias foram adotados os seguintes critérios de classificação, adaptados do método proposto por Bogaty ${ }^{12}$ : a) oclusão - lesão de $99 \%$ ou $100 \%$ em pelo menos uma artéria; b) severidade - classificado quanto ao grau de estenose nos grupos $50 \%$ a $74 \%, 75 \%$ a $90 \%$ e > $90 \%$; c) extensão - estabelecido escore segundo número de artérias comprometidas e presença de lesão em tronco de artéria coronária esquerda: estenose $>70 \%$ em artéria coronária direita $=1$ ponto; estenose $>70 \%$ em artéria coronária esquer$\mathrm{da}=1$ ponto; estenose $>70 \%$ em artéria circunflexa $=1$ ponto; estenose $>50 \%$ em tronco de artéria coronária esquerda $=2$ pontos $\mathrm{O}$ escore do paciente foi estabelecido pela soma dos pontos.

Os pacientes foram considerados hipertensos quando referiam história de hipertensão diagnosticada por seu médico assistente ou serviço médico que lhe prestasse assistência, e faziam tratamento dietético e/ou farmacológico. A presença de tabagismo foi definido como consumo de pelo menos cinco cigarros por dia, por no mínimo um ano, nos últimos cinco anos. Os pacientes diabéticos foram classificados pelo diagnóstico comprovado de diabetes mellitus. A obesidade foi definida pelo índice de Quetelet, de acordo com o National Institutes of Health Consensus Development ${ }^{17}$ : superior a 27,3 para as mulheres e maiores do que 27,8 em homens. O tempo de doença coronária correspondeu ao período entre o $1^{\circ}$ diagnóstico médico de doença coronária até o momento da inclusão no trabalho. Os limites 


\begin{tabular}{|llll|}
\hline \multicolumn{4}{|c|}{ Tabela I - Características dos pacientes estudados } \\
\hline & Grupo infarto & Grupo angina \\
\hline Idade (anos \pm desvio padrão) & $59,9 \pm 10,3$ & $57,8 \pm 10,9$ & \\
Homens (\%) & 75,8 & 47,3 & $\mathrm{p}<0,00016$ \\
Obesidade (\%) & 9,7 & 23,3 & $\mathrm{p}<0,024$ \\
Tabagismo (\%) & 46,8 & 22,5 & $\mathrm{p}<0,0006$ \\
Hipertensão arterial sistêmica (\%) & 46,8 & 18,5 & \\
Diabetes mellitus (\%) & 27,4 & 16,3 & \\
Colesterol total aumentado (\%) & 35,5 & 33,3 & \\
Sem sintomas anginosos (\%) & 18 & - & \\
Angina estável (\%) & 16 & 32,3 & $\mathrm{p}=0,018$ \\
Angina instável (\%) & 53 & 58,8 & $\mathrm{p}=0,49$ \\
Angina atípica (\%) & 1,6 & 9,2 & $\mathrm{p}=0,23$ \\
\hline
\end{tabular}

considerados normais na amostra estudada foram colesterol total $<200 \mathrm{mg} / \mathrm{dL}$, HDL colesterol $>35 \mathrm{mg} / \mathrm{dL}$, LDL colesterol $<130 \mathrm{mg} / \mathrm{dL}$, apolipoproteína A $<190 \mathrm{mg} / \mathrm{dL}$ entre os homens e $220 \mathrm{mg} / \mathrm{dL}$ entre as mulheres, apolipoproteína B $<160 \mathrm{mg} / \mathrm{dL}$ entre os homens e $150 \mathrm{mg} / \mathrm{mL}$ entre as mulheres, ferritina sérica $<262 \mathrm{mg} / \mathrm{mL}$ para homens e $155 \mathrm{mg} / \mathrm{dL}$ para mulheres. Os pontos de corte de triglicerídeos foram $<140 \mathrm{mg} / \mathrm{dL}$ entre os pacientes com até 30 anos, $<150 \mathrm{mg} /$ dL para pacientes entre 31 e 40 anos, $<160 \mathrm{mg} / \mathrm{dL}$ para pacientes entre 41 e 50 anos e $<160 \mathrm{mg} / \mathrm{dL}$ para pacientes com mais de 50 anos (segundo padrões do kit utilizado para a análise).

A análise estatística univariada foi realizada no Epiinfo versão 6, e a regressão linear múltipla bem como regressão logística foram realizadas no SPSS. Foram incluídos no modelo de regressão idade, sexo, obesidade, tabagismo, hipertensão, diabetes mellitus, colesterol total, HDL colesterol, LDL colesterol, triglicerídeos, ferritina sérica, apolipoproteínas A e B e tempo de doença coronária. O poder da amostra foi de $80 \%$ e o p alfa significativo foi $<0,05$.

\section{Resultados}

Analisando através de modelo estatístico univariado o perfil dos pacientes estudados, consideramos os dois grupos homogêneos quanto às várias características analisadas, com exceção de sexo, obesidade, tabagismo e HDL colesterol (tab. I). No grupo infarto, 29\% dos pacientes não apresentavam angina. Comparando as formas clínicas de angina em ambos os grupos, constatamos que a angina ins-

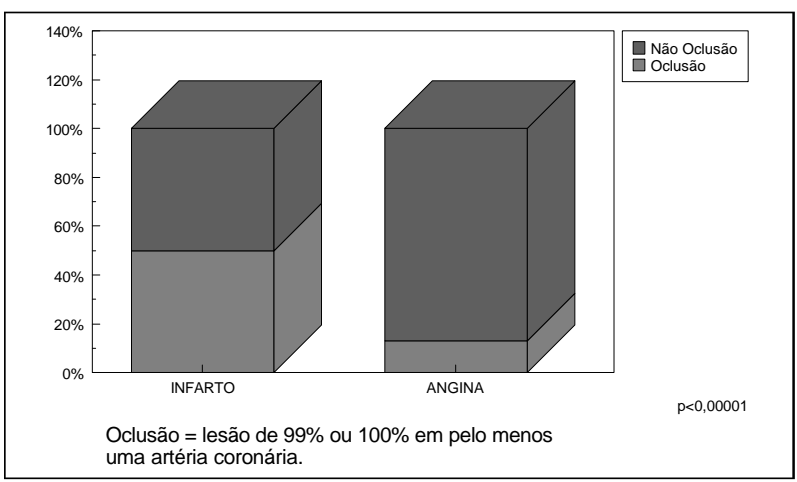

Gráfico 1 - Percentagem de pacientes com oclusão coronária.

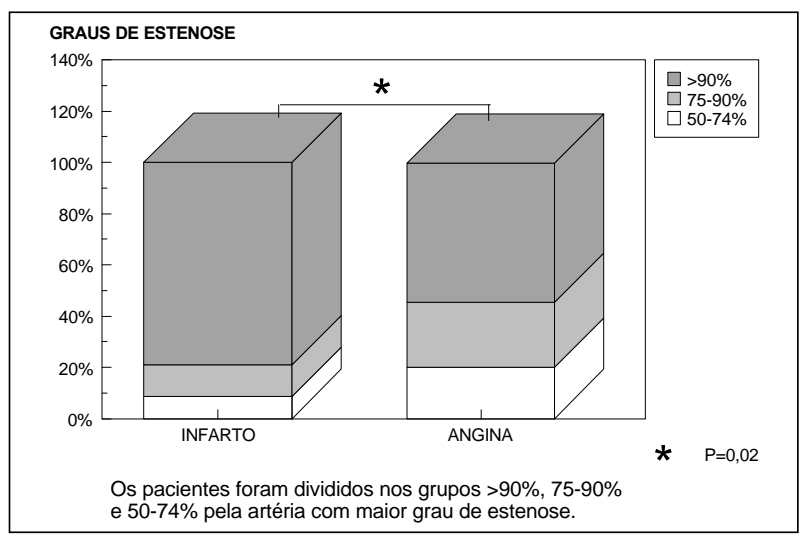

Gráfico 2 - Severidade da aterosclerose coronária.

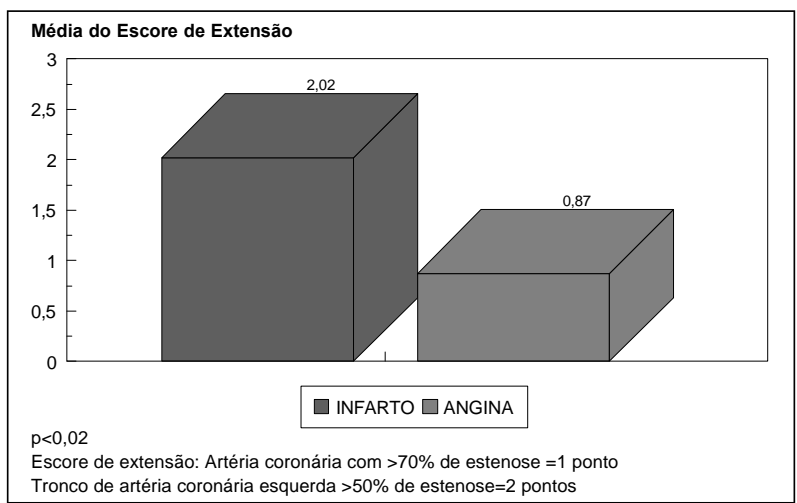

Gráfico 3 - Extensão da aterosclerose coronária.

tável ocorreu em $53 \%$ dos pacientes do grupo infarto e em $58,8 \%$ dos pacientes do grupo angina $(\mathrm{p}=0,49)$. A angina estável representou $16 \%$ no grupo infarto e $32,3 \%$ no grupo angina ( $\mathrm{p}=0,018)$, e as formas atípicas de angina $1,6 \% \mathrm{e}$ $9,2 \%$, respectivamente $(\mathrm{p}=0,23)$.

A oclusão coronária (gráfico 1) ocorreu em $50 \%$ dos pacientes do grupo infarto e em $13,2 \%$ dos pacientes do grupo angina $(\mathrm{p}<0,00001)$.

Comparando as três categorias definidas para avaliação da severidade da doença coronária (gráfico 2), os pacientes do grupo infarto (79\%) apresentaram maior número de artérias com mais de $90 \%$ de estenose em comparação com o grupo angina $(54,3 \%)(\mathrm{p}<0,02)$. Nas categorias 75 $90 \%$ e $50-74 \%$, não houve diferença estatística entre os grupos estudados.

Quando considerados os escores de extensão (gráfico 3), o grupo infarto apresentou média de 2,02, enquanto a média do grupo angina foi de $0,87(\mathrm{p}<0,02)$.

Após a análise multivariada, os resultados das variáveis oclusão, severidade e extensão não perderam a significância estatística.

Ao se analisar a relação dos fatores de risco considerados neste trabalho, nos grupos angina e infarto, controlados para extensão, severidade e oclusão, o tabagismo foi o único fator de risco independente para IM que atingiu significância estatística na amostra estudada $(p<0,05)$. No grupo infarto, $47 \%$ dos pacientes eram 
tabagistas, o que ocorreu em $22 \%$ dos pacientes do grupo angina. Quanto aos fatores de risco, a distribuição nos grupos infarto e angina foi respectivamente: diabetes mellitus - $27 \%$ e $16 \%$; HAS - $47 \%$ e $55 \%$; sexo masculino $-76 \%$ e $47 \%$; história familiar de cardiopatia isquêmica - $56 \%$ e $64 \%$; idade $>60$ anos $-47 \%$ e $39 \%$; obesidade $10 \%$ e $23 \%$. Nenhuma das diferenças entre os grupos obteve significância estatística.

\section{Discussão}

O impacto causado pela doença arterial coronária (DAC) despertou um grande interesse científico na busca de maior conhecimento da cardiopatia isquêmica, incluindo a anatomia da aterosclerose coronária. Estudos de necropsia realizados em crianças demonstraram alterações nas artérias coronárias que podem ser identificadas antes do $1^{\circ}$ ano de vida ${ }^{18-24}$. Outros estudos de necropsia, realizados posteriormente em adultos que faleceram na fase aguda do IM, demonstraram que não havia relação entre severidade da doença isquêmica, duração dos sintomas e presença de angina prévia, mas uma maior severidade e extensão da doença coronária nos pacientes com infarto transmural em relação aos que não tinham infarto transmural e nos homens em relação às mulheres ${ }^{25}$.

Com o advento da cineangiocoronariografia, tornouse possível relacionar a DAC anatômica com quadro clínico, ocorrência de IM e prognóstico, prestando informações significativas para um melhor entendimento da doença isquêmica in vivo. Os primeiros estudos que relacionaram DAC, avaliada pela cinecoronariografia, com a evolução da doença isquêmica a médio e longo prazos, demonstraram que existe uma nítida relação entre menor sobrevida e maior severidade e extensão da doença aterosclerótica nas artérias coronárias ${ }^{25,26}$. Posteriormente, novas e úteis informações foram adquiridas através da realização da cinecoronariografia em portadores de angina instável, angina estável e pacientes com IM. Os primeiros estudos realizados em portadores de angina instável demonstraram uma grande variabilidade de obstrução arterial coronária, identificandose desde obstruções únicas de $20 \%$ até lesões severas em múltiplas artérias, bem como em tronco de coronária esquerda. Foi evidenciada maior incidência de IM com alto índice de mortalidade hospitalar nos pacientes com doença aterosclerótica mais grave ${ }^{27}$. Investigações adicionais demonstraram que não existia diferença na arteriopatia coronária nos pacientes com angina instável, quando comparados àqueles com angina estável, apesar do pior prognóstico dos primeiros ${ }^{28}$.

Achados até certo ponto surpreendentes têm sido identificados pelos estudos que relacionaram, de modo evolutivo, severidade da obstrução coronária com a correspondente área de infarto, demonstrando que a artéria com maior obstrução não era a responsável pela nutrição da área infartada ${ }^{29}$. Da mesma maneira, trabalhos comparando a DAC entre pacientes com angina estável crônica e IM como $1^{\mathrm{a}}$ manifestação concluíram que a severidade e a exten- são da doença era maior no grupo com angina estável ${ }^{12}$ Outros estudos relacionaram o comprometimento arterial coronário entre pacientes com angina estável crônica, angina instável e IM como primeira manifestação, demonstrando que o comprometimento das artérias coronárias era similar entre os pacientes com angina instável e IM e mais severo nos pacientes com angina estável ${ }^{13}$.

No grupo de pacientes avaliados neste estudo, a doença aterosclerótica foi mais severa e extensa nos pacientes com IM do que nos pacientes com angina do peito.

No nosso estudo, os pacientes apresentaram o mesmo tempo de doença aterosclerótica nos dois grupos, diferindo dos trabalhos mais recentes que avaliaram o infarto como $1^{\mathrm{a}}$ manifestação, não levando em conta o tempo de doença. Devemos ressaltar que a angina instável está presente na mesma proporção em ambos os grupos, fato importante, uma vez que, em vários estudos, os pacientes com angina instável foram os que tiveram menor comprometimento coronário. Além disso, a angina estável foi encontrada em maior proporção no grupo angina $(\mathrm{p}=0,018)$ o que, de acordo com a literatura, deveria contribuir para que esse grupo tivesse mais doença coronária. Mesmo encontrando uma diferença estatisticamente significativa entre os dois grupos, se este fato estivesse alterando nossos achados poderia ser considerado um viés conservador, uma vez que, segundo dados da literatura, a presença aumentada de angina estável em um determinado grupo de pacientes deveria contribuir para que a doença aterosclerótica fosse mais severa e extensa.

Sendo assim, os dois grupos em estudo neste trabalho podem ser considerados semelhantes quanto à apresentação clínica de angina, tendo como fator diferencial apenas o fato de um grupo ter tido IM e o outro não. Desta forma, a extensão e a severidade da lesão devem estar estritamente relacionadas ao evento IM.

Nossos resultados vêm confirmar a tendência atual de implicar a instabilidade da placa aterosclerótica como principal fator envolvido no desencadeamento do IAM, uma vez que, provavelmente, a chance de um paciente ter uma placa aterosclerótica evoluindo para essa instabilidade é maior nos pacientes com um maior número de lesões.

Quanto aos fatores de risco estudados, o fumo foi o único a obter significância estatística isoladamente, quando comparados os grupos infarto e angina. Este achado vem demonstrar que a nossa população não difere de outras estudadas quanto aos malefícios do fumo na aterosclerose coronária e seus eventos clínicos ${ }^{12,13,30-33}$.

Baseando-se nos resultados e na metodologia utilizada no presente trabalho, concluímos que na população estudada, composta por habitantes do sul do país, os pacientes com IM têm maior número de artérias comprometidas, grau de estenose mais severo e número de oclusões totais do que os portadores de angina do peito sem IM.

O tabagismo mostrou ser um fator de risco significativo e independente para os pacientes acometidos de IM, quando comparados com os portadores de angina do sem IM. 


\section{Referências}

1. Jones PH, Patsch J, Gotto AM - The biochemistry of blood lipids regulation and the assessment of lipid abnormalities. In: Schlant RC, Alexander RW, O'Rourke RA, Roberts R, Sonnenblick EH, eds - Hurst's the Heart. $8^{\text {th }}$ ed. New York: McGraw-Hill, 1994: 973.

2. Ross R - The pathogenesis of atherosclerosis - an update. N Engl J Med 1996; 314: 488-500.

3. Kannel WB, Dawber TR, Kagan A, Ravatskie N, Strokes J - Factors of risck in the development of the heart disease:six year follow-up experience. The Framingham Study. Ann Intern Med 1961; 55: 33-8.

4. Giannini SD, Forti N, Issa JS, Diament J - Valor do tratamento das dislipidemias no prognóstico de pacientes com doença arterial coronária. Arq Bras Cardiol 1995; 65: 297-9.

5. Feitosa GS - Redução da colesterolemia e mortalidade. Análise crítica. Arq Bras Cardiol 1995; 65: 301-4.

6. Lindsay Jr J, Pichard AD - Acute myocardial infarction with normal coronary arteries. Am J Cardiol 1994; 54: 902-4.

7. Manfroi WC, Marques G, Goldin JR et al - Correlação entre a extensão da aterosclerose coronária e a dislipidemia. Arq Bras Cardiol 1982; 39: 15-19.

8. Manfroi WC, Gensini GG, Kelly A - Achados hemodinâmicos e cineangiocoronariográficos em pacientes acometidos de enfarte do miocárdio. Arq Bras Cardiol 1976; 29: 269-75.

9. Manfroi WC, Freitas FM, Gensini GG et al - Achados hemodinâmicos e cineangiocoronariográficos em pacientes acometidos de infarto do miocárdio. Estudo comparativo entre uma população de Syracuse, NY, USA, com outra de Porto Alegre, RS, Brasil. Arq Bras Cardiol 1980; 34: 359-62.

10. Keys A - Coronary heart disease in seven countries. Circulation 1970; 41(suppl I): I1-I211.

11. Anderson KM, Castelli WP, Levy D - Cholesterol and mortality. 30 years of follow-up from the Framingham study. JAMA 1987; 257: 2176-80.

12. Bogaty $\mathrm{P}$, Brecker SJ, White SE - Comparison of coronary angiographic findings in acute and chronic first presentation of ischemic heart disease. Circulation 1993; 87: 1938-46.

13. Cianflone D, Ciccirillo F, Buffon A - Comparison of coronary angiographic narrowing in stable angina pectoris, unstable angina pectoris and in acute myocardial infarctation. Am J Cardiol 1995; 76: 215-19.

14. Little WC, Constantinescu M, Applegate RJ et al - Can coronary angiography predict the site of a subsequent myocardial infarctation in patients with mild-tomoderate coronary artery disease. Circulation 1988; 78: 1157-66.

15. Sones FJ, Sinrey EK - Coronary arteriography. Mod Conc Cardiovasc Dis 1962; 31: 735-40.

16. Judkins MF - Selective coronary arteriography: a percutaneous transfemural technique. Radiology 1967; 89: 814-19.

17. National Institute of Health Consensus Development Conference Statement National Institute of Health Consensus Development Panel on the Health Implications of Obesity. Ann Intern Med 1985; 103(suppl 2): 1073-7.
18. Pesonen E, Norio R, Sarna S - Thickenings in the coronary arteries in infancy as an indication of genetic factors in coronary heart disease. Circulation 1975; 51: 218-25.

19. Vlodaver Z, Kahn HA, Neufeld $\mathrm{H}$ - The coronary arteries in early life in three different ethnic groups. Circulation 1969; 39: 541-6.

20. Dock $\mathrm{W}$ - The predilection of atherosclerosis for the coronary arteries. JAMA 1946; 131: 875-80.

21. Fangman RJ, Hellwig CA - Histology of coronary arteries in newborn infants. Am J Pathol 1947; 23: 901-6.

22. Neufeld HN, Wagenwoort CA, Edwards JE - Coronary arteries in fetuses, infants, juveniles and young adults. Lab Invest 1962; 11: 837-42.

23. Pesonen E - Coronary wall thickening of arteries. An analysis of the factors associates with the growth of arterial layers. Atherosclerosis 1974; 20: 173-9.

24. Wilens SL, Plair CM, Henderson D - Size of the major epicardial coronary arteries at necropsy. Relation to age, heart weight and myocardial infarction. JAMA 1966; 198: 1325-30.

25. Roberts WC, Jones AA - Quantification of coronary arterial narrowing at necropsy in acute transmural myocardial infarction. Analysis and comparison of findings in 27 patients and 22 controls. Circulation 1980; 61: 786-90.

26. Humphries JO, Kuller L, Ross RS, Friesinger GC, Page EE - Natural history of ischemic heart disease in relation to arteriographic findings. A twelve year study of 224 patients. Circulation 1974; 49: 489-97.

27. Slagle RC, Bartel AG, Behar VS, Peter R, Rosati RA, Kong Y - Natural history of angiographically documented coronary arteries disease. Circulation 1972; 44(suppl II): II-60-5.

28. Alison HW, Russell Jr RO, Mantle JA, Kouchoukos NT, Moraski RE, Rackley CE - Coronary anatomy and arteriography in patients with unstable angina pectoris. Am J Cardiol 1978; 41: 204-9.

29. Rafflenbleul W, Smith LR, Rogers WJ, Mantle JA, Rackley CE, Russell Jr RO Quantitative coronary arteriography. Coronary anatomy of patients with unstable angina pectoris reexaminated 1 year after optimal medical therapy. Am J Cardiol 1979; 43: 699-707.

30. Zimmerman FH, Cameron A, Fisher LD, Grace NG - Myocardial infarction in young adults: angiographic characterization, risk factors and prognosis. J Am Coll Cardiol 1995; 26: 654-611

31. Garrison RJ, Kannel WB, Feinleib M et al - Cigarrette smoking and HDL cholesterol. The Framingham Offspring study. Atherosclerosis 1978; 30: 17-25.

32. Brischetto CS, Connor WE, Connor SL et al - Plasma lipid and lipoprotein profiles of cigarrette smokers from randomly selected families: enhancement of hyperlipidemia and depression of high density lipoprotein. Am J Cardiol 1983; 52: 675-80.

33. Aberg A, Bergstrand R, Johannson S et al - Cessation of smoking after myocardial infarction. Effects on mortality after ten years. Br Heart J 1983; 49: 416-22. 